\title{
The Hidden Evil: Gastrointestinal Bleed and Small Bowel Obstruction Caused by Carcinoid Tumor Found on Exploratory Laparotomy
}

\author{
Parminder S. Minhas ${ }^{\text {a, c }}$, Jaspreet K. Virdia ${ }^{a}$ Richard T. Goldhahn ${ }^{b}$
}

\begin{abstract}
Intestinal carcinoid tumors are uncommon malignancies which grow slowly and rarely cause any symptoms. Small bowel tumors can, at times, cause bowel obstruction and rarely, bleeding. We present a 52-year-old male who presented to the hospital for evaluation of melena. His endoscopy and colonoscopy came back negative and patient unfortunately failed to get capsule endoscopy as an outpatient. One year later, patient presented with excruciating abdominal pain and was found to have small bowel obstruction with multiple transition points. Patient was found to have four nodular lesions in the small intestine which were found to be carcinoid tumor. Surgical resection definitely improved his outcome and patient did not need adjuvant therapy after surgery. This patient was a diagnostic challenge due to unusual presentation and negative CT scan imaging during both presentations. Carcinoid tumors are highly infiltrating tumors; hence, high degree of suspicion should be kept for earlier detection and better outcome.
\end{abstract}

Keywords: Carcinoid tumor; Small bowel; Obstruction; Bleeding; Scintigraphy

\section{Introduction}

Carcinoid tumors are rare, slow-growing tumors that originate in cells of the diffuse neuroendocrine system. They occur most frequently in tissues derived from the embryonic gut. Foregut tumors, which account for up to $25 \%$ of cases, arise in the lung, thymus, stomach, or proximal duodenum. Midgut tumors, which account for up to $50 \%$ of cases, arise in the small intestine, appendix, or proximal colon. Hindgut tumors, which

\footnotetext{
Manuscript accepted for publication June 29, 2016

aDepartment of Internal Medicine, Abington Jefferson Health, 1200 Old York Rd, Abington, PA, USA

bDepartment of Pathology, Abington Jefferson Health, 1200 Old York Rd, Abington, PA, USA

${ }^{\mathrm{c} C}$ Corresponding Author: Parminder S. Minhas, Department of Internal Medicine, Abington Jefferson Health, 1200 Old York Rd, Abington, PA, USA. Email: pminhas@abingtonhealth.org
}

doi: http://dx.doi.org/10.14740/jmc2562w account for approximately $15 \%$ of cases, arise in the distal colon or rectum [1].

As carcinoid tumors are slow growing, patients with these lesions may be asymptomatic. Some patients may complain of a long history of intermittent crampy abdominal pain, weight loss, fatigue, abdominal distention, diarrhea, or nausea and vomiting. Rarely, this primary lesion can also cause small bowel obstruction or intestinal bleeding [2,3]. Partial intestinal obstruction can be the result of an intense desmoplastic reaction characteristic of carcinoid tumors. Lower gastrointestinal bleeding can result from ulceration of the mucosa overlaying the tumor.

\section{Case Report}

We present a diagnostic dilemma case of a 52-year-old male who initially presented to the hospital with black stools and anemia. CT scan did not show any obvious mass, just nonspecific prominence of few mesenteric lymph nodes. Patient subsequently had EGD and colonoscopy, which were both negative. Patient was discharged with the instructions to follow up with gastroenterologist as an outpatient for possible capsule endoscopy. Unfortunately, patient failed to do so. One year later, patient presented to the ER with extreme abdomi-

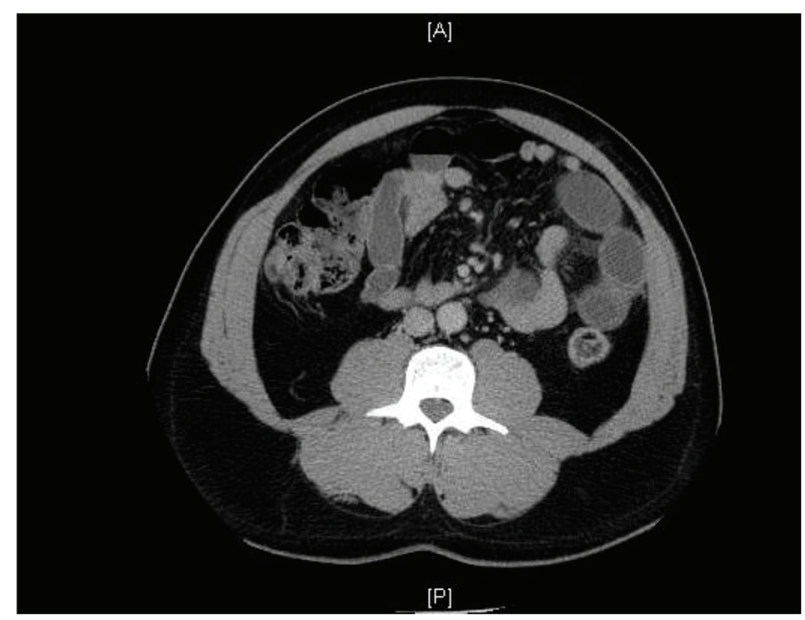

Figure 1. CT scan image of abdomen showing multiple dilated small bowel loops with several transition points. 


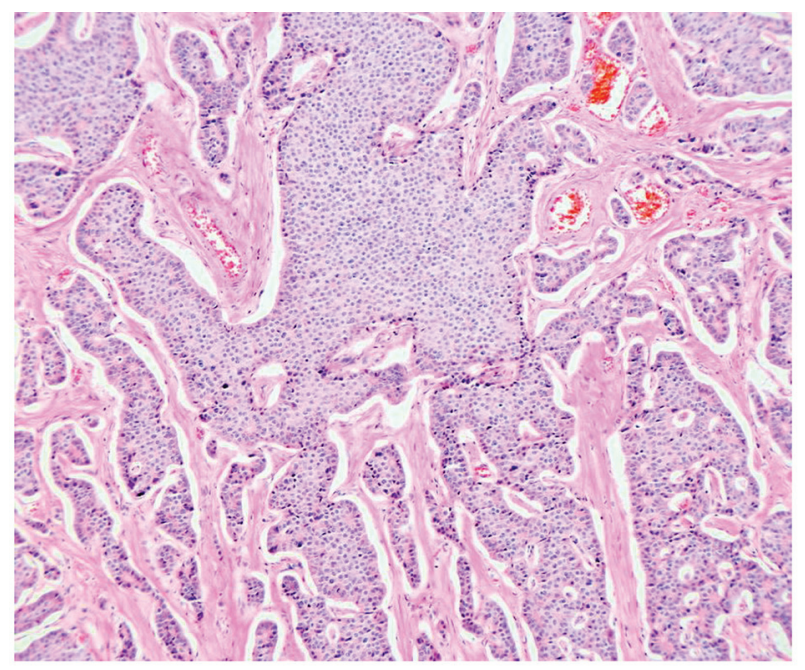

Figure 2. Histopathology. Medium magnification showing tumor pattern into the muscle.

nal pain. CT of abdomen/pelvis was obtained demonstrating multiple dilated loops of small bowel with haziness/fluid in the mesentery, submucosal edema, and several transition points, concerning for closed loop obstruction (Fig. 1). He underwent exploratory laparotomy which did not reveal a clear transition point. Four nodular areas were noted in the small bowel and resected, the largest one being $1.5 \mathrm{~cm}$. Three lymph nodes were removed. All nodules were consistent with invasive carcinoid tumor. All lesions invaded through the muscularis propria. All margins were negative. Out of three lymph nodes, one was positive for metastatic carcinoid tumor. There was also a separate segment of small bowel resected with a mesenteric lymph node that revealed marked follicular hyperplasia. All tumor and metastatic lymph node sampling stained identically with chromogranin, synaptophysin and pankeratin staining (Figs. 24). His chromogranin A level was 8 postoperatively and a $24-\mathrm{h}$ urine for 5-HIAA was within normal limits.

Postoperatively, patient made uneventful recovery and was started on clears after 3 days. Patient did not require adju-

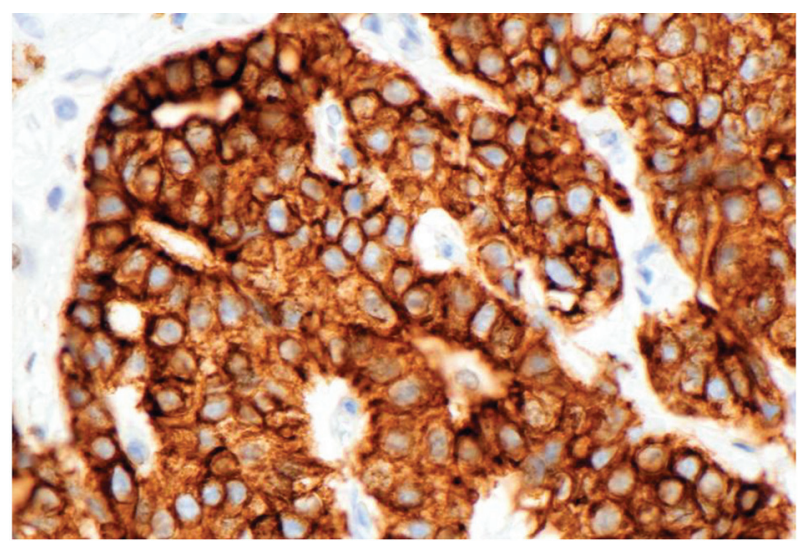

Figure 3. Slide showing the immunoperoxidase staining to pankeratin antibody, indicating the tumor's epithelial characteristics. Original magnification $\times 400$.

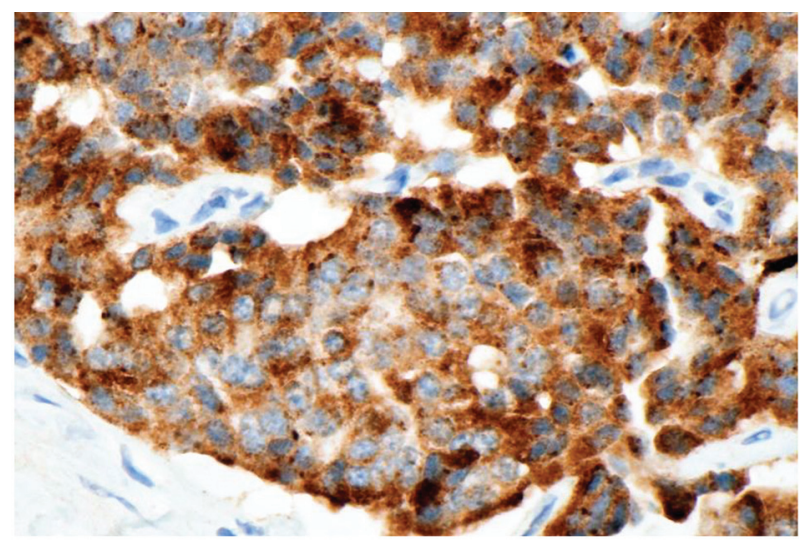

Figure 4. Slide showing the immunoperoxidase staining of the tumor cells to chromogranin antibody, indicating the neuroendocrine nature of the tumor. Original magnification $\times 400$.

vant therapy with somatostatin analogues and was asked to get surveillance CT scan every 3 months for first 2 years.

\section{Discussion}

We bring this case due to multiple reasons. First, intestinal carcinoid tumors are uncommon tumors and multiple tumors are extremely rare. Carcinoids of the small intestine account for $15.4 \%$ of all gastrointestinal carcinoids and are multicentric in $20-30 \%$ of patients $[4,5]$. Second, carcinoid tumors are mostly asymptomatic but can sometimes cause small bowel obstruction and rarely, bleeding too. Third, CT and MRI may be used for initial localization of the tumor only because both imaging techniques may miss lesions otherwise detected by 111 Inoctreotide scintigraphy; one study has shown that lesions in $50 \%$ of patients were missed, especially in lymph nodes and extrahepatic locations [6, 7]. Last but not the least, prognosis can be markedly improved by early diagnosis and surgical management. Hence, it should be considered as differential diagnosis in patients who present with bleeding with normal endoscopy and colonoscopy and patients presenting with small bowel obstruction.

\section{References}

1. Scarsbrook AF, Ganeshan A, Statham J, Thakker RV, Weaver A, Talbot D, Boardman P, et al. Anatomic and functional imaging of metastatic carcinoid tumors. Radiographics. 2007;27(2):455-477.

2. Levy AD, Sobin LH. From the archives of the AFIP: Gastrointestinal carcinoids: imaging features with clinicopathologic comparison. Radiographics. 2007;27(1):237257.

3. Akerstrom G, Hellman P, Hessman O, Osmak L. Management of midgut carcinoids. J Surg Oncol. 2005;89(3):161169.

4. Memon MA, Nelson H. Gastrointestinal carcinoid tumors: current management strategies. Dis Colon Rectum. 
1997;40(9):1101-1118.

5. Yamaguchi T, Manabe N, Tanaka S, Fukumoto A, Shimamoto M, Nakao M, Kamino D, et al. Multiple carcinoid tumors of the ileum preoperatively diagnosed by enteroscopy with the double-balloon technique. Gastrointest Endosc. 2005;62(2):315-318.

6. Modlin IM, Latich I, Zikusoka M, Kidd M, Eick G, Chan
AK. Gastrointestinal carcinoids: the evolution of diagnostic strategies. J Clin Gastroenterol. 2006;40(7):572582.

7. Shi W, Johnston CF, Buchanan KD, et al. Localization of neuroendocrine tumors with [111In] DTPA-octreotide scintigraphy (Octreoscan): a comparative study with CT and MR imaging. QJM. 1998;91 (4):295-301. 